Prof. Putnam ; 'Results of Recent Cave Exploration in the United States,' by H. C. Mercer; 'Kootenay Indian Place Names and Names of Implements,' by Prof. A. F. Chamberlain ; 'Clan System of the Pueblos,' by F. W. Hodge. A very suggestive paper by Rev. Dr. Richert on 'Character and Food.' An account of 'Finland Vapor Baths,' by Mr. H. W. Smith, and an account of certain uses in religious ceremonies of the 'Mescal Plant,' by James Mooney.

The officers elected for next year for this Section are: Vice President, W J McGee, Washington, D. C. ; Secretary, H. I. Smith, New York.

G. H. Perkins,

UNIVERSITY OF VERMONT. Secretary.

\section{THE SOCIETY FOR THE PROMOTION OF AGRICULTURAL SCIENCE.}

THE seventeenth annual meeting of the Society for the Promotion of Agricultural Science was held at Buffalo, N. Y., in the Public Library Building, on August 21st and 22d. The meeting was the most successful one of recent years. Fifteen papers, on the following subjects, were read, most of the authors being present in person:

W. R. LAzENBY. Presidential address. The Relation of Science to Agriculture.

A. D. Hopkins : On varieties of timothy and red clover.

Pollen-distributing insects observed on flowers of timothy and red clover.

V. A. MOorE: The influence of animal experimentation upon agriculture.

C. C. Georateson: Steer feeding experiments at the Kansas Experiment Station.

L. O. How ARD: A biographical sketch of Dr. C. V. Riley.

B. M. DugGar (By invitation): Sporotrichum globuliferum: White muscardine of the chinch bug economically considered.

E. A. DE SCHWEINITZ: An anti-toxic serum for hog cholera and swine plague. The production of immunity to hog cholera by means of the blood serum of immune animals.

H. L. BoLLEY: The relation of the time of feeding and the period of development, to the develop- ment of rusts and smuts in oats. Also some further experiments on potato scab.

F. D. Chester: Protective inoculation against anthrax.

H. C. IRISH (By invitation): Forcing cauliflower with lettuce and cucumbers.

W. A. Kellerman (By invitation): New experiments with fungicides for smuts of wheat and oats.

C. E. Bessey: A biographical sketch of Prof. C. L. Ingersoll.

F. WM. RANE: Electro-Horticulture: range of incandescent lamps.

L. H. Pammel and F. L. Scribner: Notes on grasses collected between Jefferson, Iowa, and Denver, Colorado.

The old board of officers, composing the following persons, was reelected for the ensuing year: President, W. R. Lazenby, Ohio State University, Columbus, O.; Secretary and Treasurer, C. S. Plumb, Purdue University, LaFayette, Ind.; third member Executive Committee, L. O. Howard, Department of Agriculture, Washington.

$$
\begin{aligned}
& \text { C. S. Plumb, } \\
& \text { Secretary. }
\end{aligned}
$$

\section{BRITISH ASSOCIATION FOR THE ADVANCE- MENT OF SCIENCE.* \\ ADDRESS BY THE PRESIDENT TO THE MATHEMATICAL AND PHYSICAL SECTION.}

THERE is a melancholy reminiscence connected with this meeting of our Section, for when the British Association last met in Liverpool the chair in Section A was occupied by Clerk-Maxwell. In the quarter of a century which has elapsed since that meeting, one of the most important advances made in our science has been the researches which, inspired by Maxwell's view of electrical action, confirmed that view, and revolutionized our conception of the processes occurring in the Electro-magnetic field. When the Association last met in Liverpool Maxwell's view was almost without supporters, to-day its opponents are fewer than its supporters then. Maxwell's theory, which is the development and extension of

*Liverpool meeting, beginning September 16, 1896. 
Faraday's, has not only affected our way of regarding the older phenomena of electricity, it has, in the hands of Hertz and others, led to the discovery of whole regions of phenomena previously undreamt of. It is sad to think that his premature death prevented him from reaping the harvest he had sown. His writings are, however, with us, and are a storehouse to which we continually turn, and never, I think, without finding something valuable and suggestive.

'Thus ye teach us day by day, Wisdom, though now far away.'

The past year has been rich in matters of interest to physicists. In it has occurred the jubilee of Lord Kelvin's tenure of the Professorship of Natural Philosophy at the University of Glasgow. Some of us were privileged to see this year at Glasgow an event unprecedented in the history of physical science in England, when congratulations to Lord Kelvin on the jubilee of his professorship were offered by people of every condition and country. Every scientific society and every scientific man is Lord Kelvin's debtor; but no society and no body of men owe him a greater debt than Section A of the British Association ; he has done more for this section than any one else, he has rarely missed its meetings, he has contributed to the section papers which will make its proceedings imperishable, and by his enthusiasm he has year by year inspired the workers of this section to renew with increased vigour their struggle to penetrate the secrets of nature. Long may we continue to receive from him the encouragement and assistance which have been so freely given for the past half century.

By the death of Sir W. R. Grove, the inventor of Grove's cell, we have lost a physicist whose name is a familiar one in every laboratory in the world. Besides the Grove cell, we owe to him the discovery of the gas battery, and a series of researches on the electrical behavior of gases, whose importance is only now beginning to be appreciated. His essay on the correlation of the physical forces had great influence in promoting that belief in the unity of the various branches of physics which is one of the characteristic features of modern and natural philosophy.

In the late Prof. Stoletow, of Moscow, we have lost the author of a series of most interesting researches on the electrical properties of gases illuminated by ultra-violet light, researches which, from their place of publication, are, I am afraid, not so well known in this country as they deserve to be.

As one who unfortunately of late years has had only too many opportunities of judging of the teaching of science in our public and secondary schools, I should like to bear testimony to the great improvement which has taken place in the teaching of physics in these schools during the past ten years. The standard attained in physics by the pupils of these schools is increasing year by year, and great credit is due to those by whose labors this improvement has been accomplished. I hope I may not be considered ungrateful if $I$ express the opinion that in the zeal and energy which is now spent in the teaching of physics in schools, there may lurk a temptation to make the pupils cover too much ground. You may by careful organization and arrangement ensure that boys shall be taken over many branches of physics in the course of a short time; it is indeed not uncommon to find boys of 17 or 18 who have compassed almost the whole range of physical subjects. But although you may increase the rate at which information is acquired, you cannot increase in anything like the same proportion the rate at which the subject is assimilated, so as to become a means of strengthening the mind and a permanent 
mental endowment when the facts have long been forgotten.

Physics can be taught so as to be a subject of the greatest possible educational value, but when it is so it is not so much because the student acquires a knowledge of. a number of interesting and important facts, as by the mental training the study affords in, as Maxwell said, 'bringing our theoretical knowledge to bear on the objects and the objects on our theoretical knowledge.' I think this training, can be got better by going very slowly through such a subject as mechanics, making the students try innumerable experiments of the simplest and, what is a matter of importance in school teaching, of the most inexpensive kind, but always endeavoring to arrive at numerical results, rather than by attempting to cover the whole range of mechanics, light, heat, sound, electricity and magnetism. I confess I regret the presence in examinations intended for school boys of many of these subjects.

I think, too, that in the teaching of physics at our universities, there is perhaps a tendency to make the course too complex and too complete. I refer especially to the training of those students who intend to become physicists. I think that after a student has been trained to take accurate observations, to be alive to those pitfalls and errors to which all experiments are liable; mischief may in some cases be done if he is kept performing elaborate experiments, the results of which are well known with the view of learning a knowledge of methods. It is not given to many to wear a load of learning lightly as a flower. With many students a load of learning, especially if it takes a long time to acquire it, is apt to crush enthusiasm. Now, there is, I think, hardly any quality more essential to success in physical investigations than enthusiasm. Any investigation in experimental physics requires a large expenditure of both time and patience; the apparatus seldom, if ever, begins by behaving as it ought; there are times when all the forces of nature, all the properties of matter, seem to be fighting against you ; the instruments behave in the most capricious way, and we appreciate Coutts Trotter's saying, that the doctrine of the constancy of nature was never discovered in a laboratory.

These difficulties have to be overcome, but it may take weeks or months to do so, and unless the student is enthusiastic, he is apt to retire disheartened from the contest. I think, therefore, that the preservation of youthful enthusiasm is one of the most important points for consideration in the training of physicists. In my opinion this can best be done by allowing the student, even before he is supposed to be acquainted with the whole of physics, to begin some original research of a simple kind under the guidance of a teacher who will encourage him and assist in the removal of difficulties. If the student once tastes the delights of the successful completion of an investigation, he is not likely to go back, and will be better equipped for investigating the secrets of nature than if, like the White Knight in 'Alice of Wonderland,' he commenced his career provided with the means of measuring or weighing every physical quantity under the sun, but with little desire or enthusiasm to have anything to do with any of them. Even for those students who intend to devote themselves to other pursuits than physical investigation, the benefits derived from original investigation as a means of general education can hardly be over-estimated, the necessity it entails of independent thought, perseverance in overcoming difficulties, and the weighing of evidence gives it an educational value which can hardly be rivalled. We have to congratulate ourselves that through the munificence of Mr. Ludwig Mond, in building and en- 
dowing a laboratory for research, the opportunities for pursuing original investigations in this country have been greatly increased.

The discovery at the end of last year by Prof. Röntgen of a new kind of radiation from a highly exhausted tube through which an electric discharge is passing, has aroused an amount of interest unprecedented in the history of physical science. The effects produced inside such a tube by the cathode rays, the bright phosphorescence of the glass; the shadows thrown by opaque objects, the deflection of the rays by a magnet, have, thanks to the researches of Crookes and Goldstein, long been familiar to us, but it is only recently that the remarkable effects which occur outside such a tube have been discovered. In 1893, Lenard, using a tube provided with a window made of a very thin plate of aluminium, found that a screen impregnated with a solution of a phosphorescent substance became luminous if placed outside the tube in the prolongation of the line from the cathode through the aluminium window. $\mathrm{He}$ also found that photographic plates placed outside the tube in this line were affected, and electrified bodies were discharged; he also obtained by these rays photographs through plates of aluminium or quartz. He found that the rays were affected by a magnet, and regarded them as the prolongations of the cathode rays. This discovery was at the end of last year followed by that of Röntgen who found that the region round the discharge tube is traversed by rays which can affect a photographic plate after passing through substances such as aluminium or cardboard, which are opaque to ordinary light; which pass from one substance to another, without any refraction, and with but little regular reflection; and which are not affected by a magnet. We may, I think, for the purposes of discussion, conveniently divide the rays occurring in or near a vacuum tube traversed by an electric current into three classes, without thereby implying that they are necessarily distinctly different in physical character. We have (1) the cathode rays inside the tube, which are deflected by a magnet; (2) the Lenard rays outside the tube, which are also deflected by a magnet; and (3) the Röntgen rays, which are not, as far as is known, deflected by a magnet. Two views are held as to the nature of the cathode rays; one view is, that they are particles of gas carrying charges of negative electricity, and moving with great, velocities which they have acquired as they travelled through the intense electric field which exists in the neighborhood of the negative electrode. The phosphorescence of the glass is on this view produced by the impact of these rapidly moving charged particles, though whether it is produced by the mechanical violence of the impact, or whether it is due to an electro-magnetic impulse produced by the sudden reversal of the velocity of the negatively charged particle-whether, in fact, it is due to mechanical or electrical causes, is an open question. This view of the constitution of the cathode rays explains in a simple way the deflection of those rays in a magnetic field, and it has lately received strong confirmation from the results of an experiment made by Perrin. Perrin placed inside the exhausted tube a cylindrical metal vessel with a small hole in it, and connected this cylinder with the leaves of a gold leaf electroscope. The cathode rays could, by means of a magnet, be guided so as either to pass into the cylinder through the aperture, or turned quite away from it. Perrin found that when the cathode rays passed into the cylinder the gold leaf of the electroscope diverged, and had a negative charge, showing that the bundle of cathode rays enclosed by the cylinder had a charge of negative electricity. Crookes had many years ago exposed a disc 
connected with a gold leaf electroscope to the bombardment of the cathode rays, and found that the disc received a slight positive charge ; with this arrangement, however, the charged particles had to give up their charges to the disc if the gold leaves of the electroscope were to be affected, and we know that it is extremely difficult, if not impossible, to get electricity out of a charged gas merely by bringing the gas in contact with a metal. Lord Kelvin's electric strainers are an example of this. It is a feature of Perrin's experiment that since it acts by induction, the indications of the electroscope are independent of the communication of the charges of electricity from the gas to the cylinder, and since the cathode rays fall on the inside of the cylinder, the electroscope would not be affected, even if there were such an effect as is produced when ultra-violet light falls upon the surface of an electro-negative metal when the metal acquires a positive charge. Since any such process cannot affect the total amount of electricity inside the cylinder, it will not affect the gold leaves of the electroscope ; in fact, Perrin's experiments prove that the cathode rays carry a charge of neg. ative electricity.

The other view held as to the constitution of the cathode rays is that they are waves in the ether. It would seem diffcult to account for the result of Perrin's experiment on this view, and also I think very difficult to account for the magnetic deflection of the rays. Let us take the case of a uniform magnetic field, the experiments which have been made on the magnetic deflection of these rays seem to make it clear that in a magnetic field which is sensibly uniform, the path of these rays is curved; now if these rays were due to ether waves, the curvature of the path would show that the velocity of propagation of these waves varied from point to point of the path. That is, the velocity of propagation of these waves is not.only affected by the magnetic field, it is affected differently at different parts of the field. But in a uniform field what is there to differentiate one part from another, so as to account for the variability of the velocity of wave propagation in such a field? This could not be accounted for by supposing that the velocity of this wave motion depended on the strength of the magnetic field, or that the magnetic field, by distorting the shape of the boundary of the negative dark space, changed the direction of the wave front, and so produced a deflection of the rays. The chief reason for supposing that the cathode rays are a species of wave motion is afforded by Lenard's discovery, that when the cathode rays in a vacuum tube fall on a thin aluminium window in the tube, rays having similar properties are observed on the side of the window outside the tube; this is readily explained on the hypothesis that the rays are a species of wave motion to which the window is partially transparent, while it is not very likely that particles of the gas in the tube could force their way through a piece of metal. This discovery of Lenard's does not, however, seem to me incompatible with the view that the cathode rays are due to negatively charged particles moving with high velocities. The space outside Lenard's tube must have been traversed by Röntgen rays, these would put the surrounding gas in a state in which a current would be readily started in the gas if any electro-motive force acted upon it. Now, though the metal window in Lenard's experiments was connected with the earth, and would, therefore, screen off from the outside of the tube any effect arising from slow electrostatic changes in the tube, it does not follow that it would be able to screen off the electrostatic effect of charged particles moving to and from the tube with very great rapidity. For in order to screen 
off electrostatic effects, there must be a definite distribution of electrification over the screen ; changes in this distribution, however, take a finite time, which depends upon the dimensions of the screen and the electrical conductivity of the material of which it is made. If the electrical changes in the tube take place at above a certain rate, the distribution of electricity on the screen will not have time to adjust itself, and the screen will cease to shield off all electrostatic effects. Thus the very rapid electrical changes which would take place if rapidly moving charged bodies were striking against the window, would give rise to electro-motive forces in the region outside the window, and would produce convection currents in the gas which has been made a conductor by the Röntgen rays. The Lenard rays would thus be analogous in character to the cathode rays, both being convective currents of electricity. Though there are some points in the behavior of these Lenard rays which do not admit of a very ready explanation from this point of view, yet the difficulties in its way seem to me considerably less than that of supposing that a wave in the ether can change its velocity when moving from point to point in a uniform magnetic field.

I now pass on to the consideration of the Röntgen rays. We are not yet acquainted with any crucial experiment which shows unmistakably that these rays are waves of transverse vibration in the ether, or that they are waves of normal vibration, or indeed that they are vibrations at all. As a working hypothesis,however,it may be worth while considering the question whether there is any property known to be possessed by these rays which is not possessed by some form or other of light. The many forms of light have in the last few months. received a noteworthy addition by the discovery of M. Becquerel of an invisible radiation, possessing many of the properties of the Rönt- gen rays, which is emitted by many fluorescent substances, and to an especially marked extent by the úranium salts. By means of this radiation, which, since it can be polarized, is unquestionably light, photographs through opaque substances similar, though not so beautiful to those obtained by means of Röntgen rays, can be taken, and, like the Röntgen rays, they cause an electrified body on which they shine to lose its charge, whether this be positive or negative.

The two respects in which the Röntgen rays differ from light is in the absence of refraction and perhaps of polarization. Let us consider the absence of refraction first. We know cases in which special rays of the spectrum pass from one substance to another without refraction ; for example, Kundt showed that gold, silver, copper allowed some rays to pass through them without bending, while other rays are bent in the wrong direction. Pflüger has lately found that the same is true for some of the aniline dyes when in a solid form. In addition to this, the theory of dispersion of light shows that there will be no bending when the frequency of the vibration is very great. I have here a curve taken from a paper by Helmholtz, which shows the relation between the refractive index and the frequency of vibration for a substance whose molecules have a natural period of vibration, and one only; the frequency of this vibration is represented by $\mathrm{OK}$ in the diagram. The refractive index increases with the frequency of the light until the latter is equal to the frequency of the natural vibration of the substance; the refractive index then diminishes, becomes less than unity, and finally approaches unity, and practically is equal to it when the frequency of the light greatly exceeds that of the natural vibration of the molecule. Helmholtz's results are obtained on the supposition that a molecule of the refracting substance consists of a pair of oppositely electrified atoms, 
and that the specific inductive capacity of the medium consists of two parts, one due to the ether, the other to the setting of the molecules along the lines of electric force.

Starting from this supposition we can easily see without mathematical analysis that the relation between the refractive index and the frequency must be of the kind indicated by the curve. Let us suppose that an electro-motive force of given amplitude acts on this mixture of molecules and ether, and start with the frequency of the external electro-motive force less than that of the free vibrations of the molecules; as the period of the force approaches that of the molecules, the effect of the force in pulling the molecules into line will increase, thus the specific inductive capacity; and therefore, the refractive index, increases with the frequency of the external force; the effect of the force on the orientation of the molecules will be greatest when the period of the force coincides with that of the molecules. As long as the frequency of the force is less than that of the molecules, the external field tends to make the molecules set so as to increase the specific inductive capacity of the mixture ; as soon, however, as the frequency of the force exceeds that of the molecules, the molecules, if there are no viscous forces, will all topple over and point so as to make the part of the specific inductive capacity due to the molecules of opposite sign to that due to the ether. Thus, for frequencies greater than that of the molecules the specific inductive capacity will be less than unity. When the frequency of the force only slightly exceeds that of the molecules, the effect of the external field on the molecules is very great, so that if there are a considerable number of molecules, the negative part of the specific inductive capacity due to the molecules may be greater than the positive part due to the ether, so that the specific inductive capacity of the mixture of molecules and ether would be negative ; no waves of this period could then travel through the medium, they would be totally reflected from the surface.

As the frequency of the force gets greater and greater, its effect in making the molecules set will get less and less, but the waves will continue to be totally reflected until the negative part of the specific inductive capacity due to the molecules is just equal to the positive part due to the ether. Here the refractive index of the mixture is zero. As the frequency of the force increases, its effect on the molecules gets less and less, so that the specific inductive capacity continually approaches that due to the ether alone, and practically coincides with it as soon as the frequency of the force is a considerable multiple of that of the molecules. In this case both the specific inductive capacity and the refractive index of the medium are the same as that of the ether and there is consequently no refraction. Thus the absence of refraction, instead of being in contradiction to the Röntgen rays, being a kind of light, is exactly what we should expect if the wave length of the light were exceedingly small.

The other objection to these rays being a kind of light is, that there is no very conclusive evidence of the existence of polarization. Numerous experiments have been made on the difference between the absorption of these rays by a pair of tourmaline plates when their axes are crossed or parallel. Many observers have failed to observe any difference at all between the absorption in the two cases. Prince Galitzine and $M$. de Karnogitsky, by a kind of cumulative method, have obtained photographs which seem to show that there is a slightly greater absorption when the axes are crossed than there is when the axes are parallel. There can, however, be no question that the effect, if it exists at all, is exceedingly small compared with the corresponding effect for visi- 
ble light. Analogy, however, leads us to expect that to get polarization effects we must use in the case of short waves, polarizers of a much finer structure than would be necessary for long ones. Thus a wire bird-cage will polarize long electrical waves, but will have no effect on visible light. Rubens and Dubois made an instrument which would polarize the infra red rays by winding very fine wires very close together on a framework; this arrangement, however, was too coarse to polarize visible light. Thus, though the structure of the tourmaline is fine enough to polarize the visible rays, it may be much too coarse to polarize the Röntgen rays if these have exceedingly small wave-lengths. As far as our knowledge of these rays extends, I think we may say that though there is no direct evidence that they are a kind of light; there are no properties of the rays which are not possessed by some variety of light.

It is clear that if the Röntgen rays are light rays, their wave-lengths are of an entirely different order to those of visible light. It is, perhaps, worth notice that on the electro-magnetic theory of light we might expect two different types of vibration if we suppose that the atoms in the molecule of the vibrating substance carried electrical charges. One set of vibrations would be due to the oscillations of the bodies carrying the charges, the other set to the oscillation of the charges on these bodies. The wave-length of the second set of vibrations would be commensurate with molecular dimensions ; can these vibrations be the Röntgen rays? If so, we should expect them to be damped with such rapidity as to resemble electrical impulses rather than sustained vibrations.

If we turn from the rays themselves to the effects they produce, we find that the rays alter the properties of the substances through which they are passing. This change is most apparent in the effects pro- duced on the electrical properties of the substances. A gas, for example, while transmitting these rays, is a conductor of electricity. It retains its conducting properties for some little time after the rays have ceased to pass through it, but $\mathrm{Mr}$. Rutherford and I have lately found that the conductivity is destroyed if a current of electricity is sent through the Röntgenized gas. The gas in this state behaves in this respect like a very dilute solution of an electrolyte. Such a solution would cease to conduct after enough electricity had been sent through it to electrolyze all the molecules of the electrolyte. When a current is passing through a gas exposed to the rays, the current destroys and the rays produce the structure which gives conductivity to the gas; when things have reached a steady state the rate of destruction by the current must equal the rate of production by the rays. The current can thus not exceed a definite value, otherwise more of the conducting gas would be destroyed than is produced.

This explains the vèry characteristic feature that in the passage of electricity through gases exposed to Röntgen rays, the current, though at first proportional to the electro-motive force, soon reaches a value where it is almost constant and independent of the electro-motive force, and we get to a state when a tenfold increase in the electro-motive force only increases the current by a few per cent. The conductivity under the Röntgen rays varies greatly from one gas to another, the halogens and their gaseous compounds, the compounds of sulphur, and mercury vapor, are among the best conductors. It is worthy of note that those gases which are the best conductors when exposed to the rays are either elements, or compounds of elements, which have in comparison with their valency very high refractive indices.

The conductivity conferred by the rays 
on a gas is not destroyed by a considerable rise in temperature ; it is, for example, not destroyed if it be sucked through metal tubing raised to a red heat. The conductivity is, however, destroyed if the gas is made to bubble through water, it is also destroyed if the gas is forced through a plug of glass wool. This last effect seems to indicate that the structure which confers conductivity on the gas is of a very coarse kind, and we get confirmation of this from the fact that a very thin layer of gas exposed to the Röntgen rays does not conduct nearly so well as a thicker one. I think we have evidence from other sources that electrical conduction is a process that requires a considerable space-a space large entough to enclose a very large number of molecules.

Thus Koller found that the specific resistances of petroleum, turpentine and distilled water, when determined from experiments made with very thin layers of these substances, was very much larger than that determined from experiments with thicker layers. Even in the case of metals there is evidence that the metal has to be of appreciable size if it is to conduct electricity. The theory of the scattering of light by small particles shows that, if we assume the truth of the electro-magnetic theory of light, the effects should be different according as the small particles are insulators or conductors. When the small particles are nonconductors, theory and experiment concur in showing that the direction of complete polarization for the scattered light is at right angles to the direction of the incident light, while if the small particles are conductors, theory indicates that the direction of complete polarization makes an angle of $60^{\circ}$ with the incident light. This result is not, however, confirmed by the experiments made by Prof. Threlfall on the scattering of light by very small particles of gold. $\mathrm{He}$ found that the gold scattered the light in just the same way as a non-conductor, giving complete polarization at right angles to the incident light. This would seem to indicate that those very finely divided metallic particles no longer acted as conductors. Thus there seems evidence that in the case of conduction through gases, through badly conducting liquids and through metals, electric conduction is a process which requires a very considerable space and aggregations of large numbers of molecules. I have not been able to find any direct experimental evidence as to whether the same is true for electrolytes. Experiments on the resistance of thin layers of electrolytes would be of considerable interest, as according to one widely accepted view of electrolysis conduction through electrolytes, so far from being effected by aggregations of molecules, takes places by means of the ion, a structure simpler than that of the molecule, so that if this represents the process of conduction, there would not seem room for the occurrence of an effect which occurs with every other kind of conduction.

In this building it is only fitting that some reference should be made to the question of the movement of the ether. You are all doubtless acquainted with the heroic attempts made by Prof.: Lodge to set the ether in motion, and how successfully the ether resisted them. It seems to be conclusively proved that a solid body in motion does not set in motion the ether at an appreciable distance outside it; so that if the ether is disturbed at all in such a case, the disturbance is not comparable with that produced by a solid moving through an incompressible fluid, but must be more analogous to that which would be produced by the motion through the liquid of a body of very open structure, such as a piece of wire netting, where the motion of the fluid only extends to a distance comparable with the diameter of the wire, and not 
with that of the piece of netting. There is another class of phenomena relating to the movement of the ether which is, I think, deserving of consideration, and that is the effect of a varying electro-magnetic field in setting the ether in motion. I do not remember to have seen it pointed out that the electro-magnetic theory of light implicitly assumes that the ether is not set in motion even when acted on by mechanical forces. On the electro-magnetic theory of light such forces do exist, and the equations used are only applicable when the ether is at rest. Consider, for example, the case of a plane electric wave travelling through the ether. We have parallel to the wave-front a varying electric polarization, which on the the ory is equivalent to a current; at right angles to this, and also in the wave-front, we have a magnetic force. Now, when a current flows through a medium in a magnetic field there is a force acting on the medium at right angles to the plane, which is parallel both to the current and to the magnetic force; there will thus be a mechanical force acting on each unit volume of the ether when transmitting an electric wave, and since this force is at right angles to the current and to the magnetic force, it will be in the direction in which the wave is propagated. In the electro-magnetic theory of light, however, we assume that this force does not set the ether in motion, as unless we made this assumption we should have to modify our equations; as the electro-magnetic equations are not the same in a moving field as in a field at rest. In fact, a complete discussion of the transmission of electro-magnetic disturbances requires a knowledge of the constitution of the ether which we do not possess. We now assume that the ether is not set in motion by an electro-magnetic wave. If we do not make this assumption we must introduce into our equation quantities representing the components of the velocity of the ether, and unless we know the constitution of the ether, so as to be able to deduce these velocities from the forces acting on it, there will be in the equations of the electro-magnetic field more unknown quantities than we have equations to determine. It is, therefore, a very essential point in electro-magnetic theory to investigate whether or not there is any motion of the ether in a varying electro-magnetic field. We have at the Cavendish Laboratory, using Prof. Lodge's arrangement of interference fringes, made some experiments to see if we could detect any movement of the ether in the neighborhood of an electric vibrator, using the spark which starts the vibrations as the source of light. The movement of the ether, if it exists, will be oscillatory, and with an undamped vibrator the average velocity would be zero; we used, therefore, a heavily damped vibrator, with which the average velocity might be expected to be finite. The experiments are not complete, but so far the results are entirely negative. We also tried by the same method to see if we could detect any movement of the ether in the neighborhood of a vacuum tube emitting Röntgen rays, but could not find any trace of such a movement. Prof. Threlfall, who independently tried the same experiment, has, I believe, arrived at the same conclusion.

Unless the ether is immovable under the mechanical forces in a varying electromagnetic field, there are a multitude of phenomena awaiting discovery. If the ether does move, then the velocity of transmission of electrical vibrations, and therefore of light, will be affected by a steady magnetic field. Such a field, even if containing nothing but ether, will behave towards light like a crystal, and the velocity of propagation will depend upon the direction of the rays. A similar result would also hold in a steady electric field. We 
may hope that experiments on these and similar points may throw some light on the properties of that medium which is universal, which plays so large a part in our explanation of physical phenomena, and of which we know so little.

\section{J. J. Thоmson.}

\section{CURRENT NOTES ON ANTHROPOLOGY.}

PATHOLOGY IN ANTHROPOLOGY.

In a note in ScIence, May 1st, I vindicated the importance of the.study, in anthropology, of pathological traits and processes. In the Revue Mensuelle de l'Ecole d'Anthropologie for July 15th is an excellent article on the same theme from Prof. Capitan. He sets forth with brevity and precision the many applications of pathology in anthropologic investigations. For instance, the diseased conditions of bones throw much light on prehistoric society; the disturbances of nutrition and reproduction solve many a problem in ethnic biology; defects in the organs of the senses explain the traits of various tribes; endemic, epidemic and hereditary diseases control the development of nations; migrations and dispersions are governed by similar causes; mental maladies are fruitful of extraordinary results in ethnic history, and so on.

But he takes a step further, a bold one, and, one must say, not a false one. "The generally received notion that humanity at large is in a healthy condition, normal and physiological, is an utter error. There is not a single individual, still less a large number, who are thoroughly sound; we always study them in a more or less diseased condition." There is no doubt this is so, and its consequences deserve far more attention than they have received.

THE CROWD AS AN ANTHROPIC UNIT.

ETHNOGRAPHERS have been accustomed to deal with the ' race,' the 'tribe' and the 'nation' as social or anthropic units; but of late it has become evident that the ' crowd,' any crowd, anywhere, anytime, is just as specialized, has as many individual traits, and is quite as active in its influence, as either of those mentioned. The 'crowd' may be in the salon of a lady of fashion, on a corner in the slums, or at a meeting of a scientific association; it will have the same peculiarities and move according to the same laws. It will act on impulse and not on reason; its intelligence is that of its most inferior members; but its powers are prompt and far-reaching. Mental suggestion and mental contagion are its favorite stimuli. It loves catch-words, symbols, colors and costumes. It prizes a badge far above a syllogism, and can be captured by the former when the latter would fall powerless.

The study of this many-headed beast has very properly come into the scope of anthropology, and the little book of Dr. Gustave Le Bon, 'Psychologie des foules' (Alcan, Paris), as well as the lectures of Prof. Bernheim, of Nancy, on 'Suggestion collective,' enable the reader to appreciate how singularly the folly of the mass obscures the wisdom of the individual.

\section{RECENT CRANIOLOGICAL STUDIES.}

Dr. Rudolf Martin, already familiar to Americanists by his somatologic writings on the natives of Tierra del Fuego, has lately published in the quarterly journal of the Naturforschende Gesellschaft, of Zürich, an article on 'Old Patagonian Skulls.' The crania, twelve in number, were obtained from the left bank of the Rio Negro, about fifty kilometers above its mouth. He subjects them to a searching scrutiny and an analysis of their dimensions. They do not seem to show marked traits of degeneration. In form they are brachycephalic and prognathic, with prominent cheek bones. Two full-plate illustrations 